\title{
Limits in the characteristic function description of non-Lindblad-type open quantum systems
}

\author{
Sabrina Maniscalco ${ }^{1,2}$ \\ ${ }^{1}$ School of Pure and Applied Physics, University of KwaZulu-Natal, Durban 4041, South Africd* \\ ${ }^{2}$ INFM, MIUR and Dipartimento di Scienze Fisiche ed Astronomiche \\ dell'Università di Palermo, via Archirafi 36, 90123 Palermo, Italy
}

(Dated: September 14, 2018)

\begin{abstract}
In this paper I investigate the usability of the characteristic functions for the description of the dynamics of open quantum systems focussing on non-Lindblad-type master equations. I consider, as an example, a non-Markovian generalized master equation containing a memory kernel which may lead to nonphysical time evolutions characterized by negative values of the density matrix diagonal elements [S.M. Barnett and S. Stenholm, Phys. Rev. A 64, 033808 (2001)]. The main result of the paper is to demonstrate that there exist situations in which the symmetrically ordered characteristic function is perfectly well defined while the corresponding density matrix loses positivity. Therefore nonphysical situations may not show up in the characteristic function. As a consequence, the characteristic function cannot be considered an alternative complete description of the non-Lindblad dynamics.

PACS numbers: 03.65.Yz, 03.65.Ta
\end{abstract}

The theory of open quantum systems describes the interaction of a quantum system with its environment 1 ]. Although many physical systems may be considered under certain conditions quasi-closed for certain intervals of time, no quantum system may be seen as completely isolated from its surroundings. The unavoidable interaction between the system and its environment leads to the phenomena of decoherence and dissipation [2].

The description of the dynamics of open systems has recently attracted much of attention for mainly two reasons. On the one hand environment induced decoherence due to the establishment of entanglement between the system and the environment is one of the key issues of the quantum measurement theory 2]. On the other hand, the system-environment interaction seems to be the major limiting factor in the realization of quantum devices necessary for the new quantum technologies, e.g. quantum computation [3].

The study of the dynamics of an open system is, in general, a very difficult task. Usually, even if one is interested in the dynamics of the system only, the influence of the typically infinite environmental degrees of freedom, makes it impossible to solve exactly the equations of motion for the observables of interest. For this reason, the standard description of open systems rely on a number of approximations which allow to derive a master equation for the reduced density matrix of the system. The two most common approximations are the weak coupling approximation, valid when the interaction between system and environment is sufficiently weak, and the Markov approximation, relying on the assumption that the characteristic times of the system are much larger than those of the environment [4].

Generally, when these approximations are satisfied, the master equation for the reduced density matrix may be written in the so-called Lindblad form, which is the only possible form of first-order linear differential equation, for a completely positive dynamical semigroup having bounded generator [5, 6]. The Lindblad master equation, however, is valid as long as the weak coupling and Markov approximation hold. While these assumptions are often well justified in quantum optics, in many solid-state systems, i.e. photonic band-gap materials and quantum dots, the Markov approximation does not hold 7]. Similarly, the reservoir interacting with a single mode cavity in atom lasers is strongly non-Markovian [8]. NonMarkovian generalized master equations usually ar not of Lindblad type.

It is worth noticing that there exist also Markovian systems described by master equations which cannot be cast in the Lindblad form [9, 10. An important problem in the description of open quantum systems whose master equations are not in the Lindblad form is that their dynamical map needs not be completely positive [1], and this may lead to physical inconsistency. It may even happen that the positivity condition of the density matrix during the time evolution, a condition less restrictive then complete positivity but necessary to guarantee the probabilistic interpretation of the density matrix, breaks down.

For the sake of completeness, let me underline that complete positivity is a necessary requirement for a consistent physical description of open quantum systems whenever factorized initial conditions for the system and the reservoir are assumed, i.e. $\hat{\rho}_{\mathrm{T}}(0)=\hat{\rho}(0) \otimes \hat{\rho}_{\mathrm{E}}(0)$, with $\hat{\rho}_{\mathrm{T}}(0), \hat{\rho}(0), \hat{\rho}_{\mathrm{E}}(0)$, initial density matrices of the total system, of the reduced system of interest and of the environment, respectively. Most of the derivations of master equations found in the literature rely on this assumption. However, when correlations are present at the ini- 
tial time, acceptable quantum dynamics which are not completely positive may exist 13. In the following I will focus on the case of factorized initial conditions for which the dynamical map must be completely positive.

When working with non-Markovian generalized master equations, or with master equations which are not in the Lindblad form, it is of crucial importance to establish conditions under which the density matrix preserves positivity and complete positivity during the time evolution. In most of the cases these conditions are given in terms of the density matrix elements at time $t$, and therefore require the knowledge of the analytic solution of the nonMarkovian master equation. In Ref. [14], conditions for complete positivity in terms of the memory kernel are presented for a class of generalized master equations. To the best of the author's knowledge, however, a criterion analogous to the Lindblad one for general master equations with memory has not yet been formulated.

Very useful tools for the description of the dynamics of paradigmatic open quantum systems such as the damped harmonic oscillator (quantized mode of the electromagnetic field, motion of a trapped ion) or the quantum Brownian particle, are the characteristic functions and the quasi-probability distribution functions. Both of them contain all the information necessary to reconstruct the density matrix, and therefore they have been considered up to now "alternative complete descriptions of the dynamics " 15, 16]. However, the characteristic function can be considered an alternative complete description of the dynamics if and only if it is equivalent to the density matrix. I will prove in the following that, contrarily to what has been believed until now, the characteristic function and the density matrix descriptions of the dynamics cannot be considered equivalent. To the best of the author's knowledge this is the first time that the equivalence between the characteristic function and the density matrix is questioned, and an example pointing out the non-equivalence between these two approaches is presented.

Let me begin by recalling the definition of the $p$ ordered characteristic functions

$$
\begin{aligned}
\chi(\xi, p) & =\operatorname{Tr}[\hat{\rho} \hat{D}(\xi)] \exp \left(p|\xi|^{2} / 2\right) \\
& \equiv \operatorname{Tr}\left[\hat{\rho} \exp \left(\xi \hat{a}^{\dagger}-\xi^{*} \hat{a}\right)\right] \exp \left(p|\xi|^{2} / 2\right),
\end{aligned}
$$

where $\hat{D}(\xi)$ is the Glauber displacement operator and $\hat{a}\left(\hat{a}^{\dagger}\right)$ is the annihilation (creation) operator of the quantum harmonic oscillator. In the previous equation, the parameter $p$ assumes the values $p=1,0,-1$ in correspondence to normal, symmetric and antinormal ordering of the creation and annihilation operators. The two-dimensional Fourier transform of $\chi(\xi, p)$ gives the Glauber-Sudarshan $P$-representation for $p=1$, the Wigner function for $p=0$, and the Husimi $Q$-function for $p=-1$ [15.
In what follows I will focus on the $p=0$ characteristic function $\chi(\xi, p=0) \equiv \chi(\xi)$, known as simmetrically ordered characteristic function (SCF) or quantum characteristic function. Having in mind Eq. (1) it is straightforward to prove that the result obtained in this paper for the simmetrically ordered characteristic function also applies to the other two characteristic functions.

The SCF is always defined and it is, in general, a complex-valued function satisfying the following properties:

$$
\chi(\xi=0)=1 ; \quad|\chi(\xi)| \leq 1 .
$$

The first of the two properties is a consequence of the fact that $\operatorname{Tr}[\hat{\rho}]=1$, while the second stems from the fact that $\chi(\xi)$ is the expectation value of the displacement operator $\hat{D}(\xi)$ which is unitary, and therefore the magnitude of its eigenvalues is unity. One of the advantages of using the simmetrically ordered characteristic function is that the analytic expression for the mean values of many observables of interest may be calculated easily by means of the relation

$$
\left\langle a^{\dagger m} a^{n}\right\rangle=\left.\left(\frac{d}{d \xi}\right)^{m}\left(-\frac{d}{d \xi^{*}}\right)^{n} \chi(\xi)\right|_{\xi=0} .
$$

In the literature the characteristic functions have been extensively used to study the dynamics of both Markovian [15, 16, 17] and non-Markovian 9, 18, 19, 20, 21, 22. open systems. It has also been shown that characteristic functions may be used to establish observable conditions of nonclassicality for the states of the quantized electromagnetic field 23]. In what follows I will show that there exist situations in which the characteristic function description of an open quantum system may lead to problems.

Let me consider the non-Markovian dynamics of a harmonic oscillator interacting with a zero temperature reservoir. I consider one of the most popular phenomenological model for this systems, involving a memory kernel [1, 12, 14],

$$
\frac{d \hat{\rho}(t)}{d t}=\int_{0}^{t} K\left(t-t^{\prime}\right) \mathcal{L} \hat{\rho}\left(t^{\prime}\right) d t^{\prime}
$$

where $K\left(t-t^{\prime}\right)$ is the memory kernel and the Liouvillian operator $\mathcal{L}$ is given by

$$
\mathcal{L} \hat{\rho}=2 \hat{a} \hat{\rho} \hat{a}^{\dagger}-\hat{a}^{\dagger} \hat{a} \hat{\rho}-\hat{\rho} \hat{a}^{\dagger} \hat{a} .
$$

By applying the rules

$$
\begin{aligned}
& \hat{\rho} \rightarrow \chi(\xi), \hat{a} \hat{\rho} \rightarrow\left(-\frac{d}{d \xi^{*}}-\frac{\xi}{2}\right), \hat{a}^{\dagger} \hat{\rho} \rightarrow\left(\frac{d}{d \xi}-\frac{\xi^{*}}{2}\right), \\
& \hat{\rho} \hat{a} \rightarrow\left(-\frac{d}{d \xi^{*}}+\frac{\xi}{2}\right), \hat{\rho} \hat{a}^{\dagger} \rightarrow\left(\frac{d}{d \xi}+\frac{\xi^{*}}{2}\right),
\end{aligned}
$$


one may derive, from the master equation (4), the corresponding integro-differential equation for $\chi(\xi)$

$$
\begin{aligned}
\frac{\partial \chi(\xi, t)}{\partial t} & =\int_{0}^{t} K\left(t-t^{\prime}\right) \times \\
& \times\left[-\left(\xi \frac{\partial}{\partial \xi}+\xi^{*} \frac{\partial}{\partial \xi^{*}}\right)-|\xi|^{2}\right] \chi\left(\xi, t^{\prime}\right) d t^{\prime} .
\end{aligned}
$$

Following a method developed in 24] for non-Markovian Fokker-Plank equations, a formal solution of this integrodifferential equation may be obtained in form of an integral decomposition involving the solution of the corresponding Markovian problem, which is known in the literature (see, e.g., 15]).

Let me focus on the case of a memory kernel of exponential type

$$
K\left(t-t^{\prime}\right)=g^{2} e^{-\gamma\left|t-t^{\prime}\right|},
$$

with $g$ coupling strength and $\gamma$ decay constant of the system-reservoir correlations. I consider, as initial state, a Fock state $|n\rangle$, with $|n\rangle$ being the eigenstates of the quantum number operator $\hat{n}=\hat{a}^{\dagger} \hat{a}$. The corresponding SCF reads as follows

$$
\chi_{n}(\xi)=L_{n}\left(|\xi|^{2}\right) e^{-|\xi|^{2} / 2},
$$

with $L_{n}\left(|\xi|^{2}\right)$ the Laguerre polynomial of order $n$. For $n=1$, e.g., the initial simmetrically ordered characteristic function is $\chi(\xi, 0)=\left(1-|\xi|^{2}\right) e^{-|\xi|^{2} / 2}$, and it is easy to verify by direct substitution that

$$
\chi(\xi, t)=\left[1-|\xi|^{2} e^{-\gamma t / 2}\left(\cos \Omega t+\frac{\gamma}{2 \Omega} \sin \Omega t\right)\right] e^{-|\xi|^{2} / 2},
$$

is a solution of Eq. (17), with $\Omega=\sqrt{2 g^{2}-(\gamma / 2)^{2}}$. Figure 1 shows the absolute value of the simmetrically ordered characteristic function, as given by Eq. (10), as a function of $|\xi|^{2}$ at different time instants and for $g / \gamma=1$. Looking at Eq. (10) it is easy to verify that, for the parameters considered in the example, the SCF is always defined and it satisfies at all times and for each vale of $\xi$ the conditions given by Eqs. (2), as one can also see clearly from the figure.

Once the time evolution of the simmetrically ordered characteristic function is known one can always reconstruct the density matrix at all times, since $\hat{\rho}(t)$ can be obtained from $\chi(\xi, t)$ by using the relation

$$
\hat{\rho}(t)=\frac{1}{2 \pi} \int \chi(\xi, t) \hat{D}(\xi) d \xi d \xi^{*} .
$$

From this equation, keeping in mind that the diagonal elements of the Glauber displacement operator are given by

$$
\langle n|\hat{D}(\xi)| n\rangle=L_{n}\left(|\xi|^{2}\right) e^{-|\xi|^{2} / 2}
$$

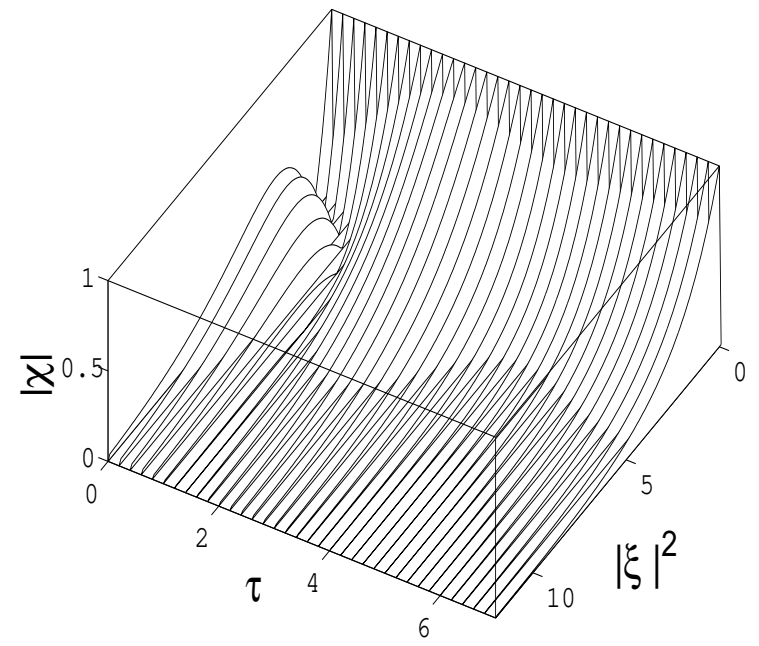

FIG. 1: Behavior of $\left|\chi\left(|\xi|^{2}\right)\right|$, as given by Eq. (10), at different times instants in the interval $0<\tau<0$. The initial state is $|n=1\rangle$ and $g / \gamma=1$.

and that $L_{1}\left(|\xi|^{2}\right)=1-|\xi|^{2}$, one gets

$$
\begin{aligned}
\rho_{11}(t) & \equiv\langle n=1|\hat{\rho}(t)| n=1\rangle \\
& =e^{-\gamma t / 2}\left(\cos \Omega t+\frac{\gamma}{2 \Omega} \sin \Omega t\right) .
\end{aligned}
$$

The quantity $\rho_{11}(t)$ describes the decay of the population of the initial state $|n=1\rangle$ due to the interaction with a zero-temperature reservoir. For $t \rightarrow \infty, \hat{\rho}_{11} \rightarrow 0$ and $\hat{\rho}_{00} \rightarrow 1$, indicating that, due to dissipation, the state of the oscillator passes from the initial excited Fock state $|n=1\rangle$ to the ground state. A close look to Eq. (13), however, shows that the positivity of the density matrix is clearly violated since $\hat{\rho}_{11}(t)<0$ for some intervals of time. This situation has been discussed in detail by Barnett and Stenholm in [12], where the risks of an apparently physically well grounded memory kernel, as the exponential one given by Eq. (8), were carefully analyzed. The loss of positivity shows up for strong or intermediate coupling regime $n g^{2} / \gamma \geq 1 / 8[12]$ alerting us about the fact that the dynamics of the system, for these values of the parameters, is unphysical.

It is worth stressing, however, that if one describes the time evolution by using the simmetrically ordered characteristic function, one does not realize that the equations of motion lose physical sense because this function, contrarily to the density matrix which violates one of its defining conditions (positivity), continues to verify at all times the conditions given by Eqs. (2). Hence for nonLindblad cases, when both the complete positivity and the positivity conditions may be violated, unphysical situations, such as the negativity of the density matrix, may not show up in the dynamics of the SCF.

A careful analysis of Eq. (10) shows that only for values of the ratio $g / \gamma$ such that, for certain intervals of time, $\rho_{11}(t)<-1 / 2$ then the second of the conditions 
given in Eqs. (2) is violated for $|\xi|^{2} \ll 1$. In general, however, there is no correspondence between the loss of the positivity condition, and therefore of complete positivity, and the violation of one of the conditions defining the simmetrically ordered characteristic function. Stated another way the problem is the following. The fact that $\hat{D}(\xi)$ is an unitary operator, together with the properties that $\hat{\rho}$ is a positive (then Hermitian) trace-class operator with trace 1, imply that $|\chi(\xi)| \leq 1$, i.e. the second of Eq. (2). This is, however, only a necessary condition, indeed I have shown in the paper that there exist situations in correspondence of which the density matrix is not positive but still $|\chi(\xi)| \leq 1$. The crucial question is therefore, which is the additional condition to be imposed on the SCF to ensure that the operator $\hat{\rho}$, defined through Eq. (11), is a positive trace-class operator with trace 1 ? The answer to this question is very important since it would allow to use safely the SCF in non-Lindblad cases. Until when this condition is not found, one cannot claim that the density matrix and the SCF are equivalent descriptions of the dynamics, as the example given in this paper clearly indicates. The derivation of the condition to be imposed on the simmetrically ordered characteristic function to make it a useful tool in the description of non-Lindblad type situations will be the object of further study. However, presently, it seems to the author that this question does not have a simple answer.

Let me conclude considering the behaviour of the Wigner function. The Wigner function is the twodimensional Fourier transform of the simmetrically ordered characteristic function

$$
W(\alpha)=\frac{1}{\pi^{2}} \int_{-\infty}^{\infty} d \xi d \xi^{*} \chi(\xi) e^{\alpha \xi^{*}-\alpha^{*} \xi} .
$$

The Wigner function is a real valued function satisfying the condition $|W(\alpha)| \leq 2 / \pi$. Inserting Eq. (10) into Eq. (14) we get

$$
W(\alpha, t)=\frac{2}{\pi} e^{-2|\alpha|^{2}}\left[1+2\left(2|\alpha|^{2}-1\right) \rho_{11}(t)\right],
$$

with $\rho_{11}(t)$ given by Eq. (13). From the previous equation one may verify that whenever $0 \leq \rho_{11}(t) \leq 1$, then $|W(\alpha)| \leq 2 / \pi$, but the former inequality is immediately violated in correspondence to a violation of the positivity condition, in our example when $\rho_{11}(t)$ becomes negative.

It is worth stressing the difference between the Wigner function, which is the Fourier transform of the SCF, and the simmetrically ordered characteristic function itself. While the first one violates one of its defining conditions when the density matrix loses positivity, the second one does not. In this sense it seems that the simmetrically ordered characteristic function has less "physical meaning"than the density matrix or the Wigner function. It is worth noticing that, as a consequence of the lack of a condition equivalent to the positivity of the density matrix, each time one deals with non-Lindblad dynamics one can use the SCF only if it is possible to derive the corresponding density matrix, by means of Eq. (11), and check its positivity. In this paper I considered a rather easy example of the dynamics for which both the density matrix and the SCF solutions have simple analytic expressions. In general, however, it is not obvious that, once the solution of the Fokker-Planck equation for the $\mathrm{SCF}$ is known, one is also able to derive a useful expression for the density matrix necessary to check the positivity condition. This fact strongly limits the usability of the SCF for the study of non-Lindblad dynamics. For this reason new necessary and sufficient conditions establishing the equivalence between the SCF and the density matrix are highly desirable. Moreover, the answer to the open question posed in this paper about the identification of a condition on the SCF correspondent to positivity of the density matrix would shed light on the physically meaningful ingredient allowing to consider the description of an open quantum system via the SCF as a complete description of its dynamics.

This work has been supported by the European Union's Transfer of Knowledge project CAMEL (Grant No. MTKD-CT-2004-014427) and by the Italian National Foundation Angelo Della Riccia. The author gratefully acknowledges Antonino Messina, Francesco Petruccione, Jyrki Piilo, and Stig Stenholm for the useful comments and suggestions about the paper.

* Electronic address: maniscalco@ukzn.ac.za

[1] H.-P. Breuer and F. Petruccione, The Theory of Open Quantum systems (Oxford University Press, 2002).

[2] E. Joos, H.-D. Zeh, C. Kiefer, D. Giulini, J. Kupsch, and I.-O. Stamatescu, Decoherence and the Appearence of a Classical World in Quantum Theory, 2nd ed. (SpringerVerlag, Berlin, 2003).

[3] M. A. Nielsen and I. L. Chuang, Quantum Computation and Quantum Information (Cambridge University Press, Cambridge, England, 2000).

[4] R. Alicki and K. Lendi, Quantum Dynamical Semigroups and Applications (Springer-Verlag, Berlin, 1987).

[5] G. Lindblad, Commun. Math. Phys. 48, 119 (1976).

[6] V. Gorini, A. Kossakowski, and E.C.G. Sudarshan, J. Math. Phys. 17, 821 (1976).

[7] S. John and T. Quang, Phys. Rev. Lett. 78, 1888 (1997); T. Quang, M. Woldeyohannes, S. John, and G. S. Agarwal, Phys. Rev. Lett. 79, 5238 (1997).

[8] J.J. Hope, G.M. Moy, M.J. Collett, and C.M. Savage, Phys. Rev. A 61, 023603 (2000).

[9] W.G. Unruh and W.H. Zurek, Phys. Rev. D 40, 1071 (1989).

[10] W.J. Munro and C.W. Gardiner, Phys. Rev. A 53, 2633 (1986).

[11] K. Kraus, States, Effects and Operations, Fundamental Notions of Quantum Theory (Academic, Berlin, 1983).

[12] S.M. Barnett and S. Stenholm, Phys. Rev. A 64, 033808 (2001).

[13] P. Pechukas, Phys. Rev. Lett. 73, 1060 (1994); R. Alicki, 
ibid. 75, 3020 (1995); P. Pechukas, ibid. 75, 3021 (1995).

[14] A.A. Budini, Phys. Rev. A 69, 042107 (2004).

[15] S.M. Barnett and P.M. Radmore Methods in Theoretical Quantum Optics (Oxford University Press, Oxford, 1997).

[16] L. Mandel and E. Wolf, Optical Coherence and Quantum Optics (Cambridge University Press, Cambridge, 1995).

[17] V. Giovannetti et al., Phys. Rev. A 70, 032315 (2004).

[18] A. Isar et al., Int. J. Mod. Phys. E, Vol. 3, No 2, 635 (1994).

[19] W.T. Strunz and F. Haake, Phys. Rev. A 67, 022102 (2003).
[20] F. Intravaia, S. Maniscalco, and A. Messina, Phys. Rev. A 67, 042108 (2003).

[21] S. Maniscalco, J. Piilo, F. Intravaia, F. Petruccione and A. Messina, Phys. Rev. A 69, 052101 (2004).

[22] S. Maniscalco, J. Piilo, F. Intravaia, F. Petruccione and A. Messina, Phys. Rev. A 70, 032113 (2004).

[23] W. Vogel, Phys. Rev. Lett. 84, 1849 (2000); Th. Richter and W. Vogel, ibid. 89, 283601 (2002); E. Shchukin, Th. Richter, and W. Vogel, Phys. Rev. A 71, 011802(R) (2005).

[24] I.M. Sokolov, Phys. Rev. E 66, 041101 (2002). 